\title{
A Holistic Modeling Approach to Heavy-Ion Physics
}

\author{
Evan Sangaline* \\ National Superconducting Cyclotron Laboratory, \\ Michigan State University \\ E-mail: esangaline@gmail.com
}

\begin{abstract}
Hydrodynamic-based transport models are used to extract posterior distributions for the viscosity of the Quark-Gluon Plasma (QGP), the nuclear equation of state, and other physically meaningful quantities. These distributions are found by computing the likelihood of the model outputs in relation to a variety of experimental measurements from relativistic heavy-ion collisions at both the Relativistic Heavy Ion Collider (RHIC) and the Large Hadron Collider (LHC). This holistic approach offers the ability to make rigorous and robust statistical inferences about the equation of state of Quantum Chromodynamic (QCD) matter..
\end{abstract}

9th International Workshop on Critical Point and Onset of Deconfinement

17-21 November, 2014

ZiF (Center of Interdisciplinary Research), University of Bielefeld, Germany

\footnotetext{
* Speaker.
} 


\section{Introduction}

Relativistic heavy-ion collisions are a unique tool for probing the physics of Quantum Chromodynamic (QCD) matter at energy densities far exceeding that of cold nuclear matter. A broad range of measurements have been made which provide strong evidence that partonic, rather than hadronic, degrees of freedom are dominant at these energy densities [1, 2]. Additionally, lattice QCD calculations have shown a change in the degrees of freedom in the temperature range of 150 to $200 \mathrm{MeV}$ [3] as well as a melting of the scalar quark condensate [4] and a minimum in the speed of sound [5]. Experimental progress in constraining the equation of state in this transition region has, in contrast, been much less rigorously quantitative.

The semi-quantitative nature of previous experimental inferences about the QCD equation of state has not been due to a lack of sensitivity in experimental measurements. On the contrary, it has been shown that mean transverse momentum [6], azimuthally anisotropic flow [7, 8], and particle multiplicity measurements [9] are all sensitive to the equation of state and have been used for the purpose of constraining it. Additionally, femtoscopic measurements have largely ruled out the possibility of a very stiff equation of state [10]. Despite progress along these lines in constraining the equation of state, a more quantitative determination has remained elusive.

The field has largely converged on the use of models consisting of viscous relativistic hydrodynamics above a temperature of $\sim 165 \mathrm{MeV}$ [11] and microscopic binary collision models of the lower temperature hadronic stage in describing the bulk behavior in collisions. Despite the fact that a model of this nature encompasses the complicated interdependencies between the numerous observables and the underlying parameters, making quantitative inferences about the parameters has remained difficult due to the computational expense of the model. This has led to a proliferation of analyses in which the majority of parameters are fixed based on assumptions about the physics and then the remaining subset of parameters are varied and the observables compared to experimental measurements. This sort of approach tends to overestimate the significance of inferences and gives a largely qualitative picture of the interdependencies because the fixing of parameters dramatically alters the full likelihood distribution over parameter space given the available experimental measurements.

The work presented in these proceedings focuses on statistical inferences made in a high dimensional parameter space. The key development that makes these sort of inferences possible is the use of Gaussian-process model emulation, allowing our collision model to be evaluated at a high enough rate to employ well established Markov Chain Monte Carlo (MCMC) techniques to map out the likelihood distribution of a high dimensional parameter space given a variety of experimental results. This parameter space allows for a wide variety of possible equations of state which are in turn rigorously constrained by this analysis. The comparison of this extracted equation of state with that from lattice calculations provides important insight into the statement that the matter created in heavy-ion collisions behaves like an equilibrated Quark-Gluon Plasma (QGP).

\section{Methodology}

We focus our analysis on a set of experimental measurements made at the Relativistic Heavy Ion Collider (RHIC) and the Large Hadron Collider (LHC) consisting of $\mathrm{Au}+\mathrm{Au}$ collisions at 
$\sqrt{s_{N N}}=200 \mathrm{GeV}$ and $\mathrm{Pb}+\mathrm{Pb}$ collisions at $\sqrt{s_{N N}}=2.76 \mathrm{TeV}$ respectively. We consider 15 observables at each of these energies relating to spectra, elliptic flow, and femtoscopic source size. The mean transverse momentum, $\left\langle p_{T}\right\rangle$, of pions, kaons, and protons as well as the yield of pions were included in $0-5 \%$ and $20-30 \%$ centrality bins in order to capture the predominant features of the spectral shape. The femtoscopic source sizes, $R_{\text {out }}, R_{\text {long }}$, and $R_{\text {side }}$, averaged over the experimental momentum ranges were also included in these same centrality bins. Finally, the elliptic flow, $v_{2}\{2\}=\sqrt{\left\langle\cos 2\left(\phi_{1}-\phi_{2}\right)\right\rangle}$, was included in only the $20-30 \%$ bin. This bin was chosen to minimize the bias introduced by using smooth initial conditions and the $v_{2}$ values from the model were scaled up by a factor of 1.10 to account for this based on a comparison of the eccentricity in smooth and fluctuating initial condition models.

To make inferences from these experimental measurements, we employ a model that utilizes $2+1$ dimensional relativistic viscous hydrodynamics based on the Israel-Stewart formalism for the early evolution of the system. At a temperature of $T=165 \mathrm{MeV}$ the evolution switches to a microscopic hadronic model. The details of this model are identical to those given in [12]. The principle advancement over that previous work is that the equation of state is now considered as an unknown component of the model so that it can be constrained within a Bayesian framework. The total number of free parameters in the model, including those related to the parameterization of the equation of state, is 14 .

The equation of state was parameterized in such a way that it matches that of a hadronic gas at $T=165 \mathrm{MeV}$. At the collision energies of $\sqrt{s_{N N}} \geq 200 \mathrm{GeV}$ that are being considered we make the assumption that the baryon chemical potential is approximately zero and so the equation of state can be expressed as a function of a single variable such as the energy density $\varepsilon$. The speed of sound squared at temperatures above $165 \mathrm{MeV}$ was parameterized as

$$
c_{S}^{2}(\varepsilon)=c_{s}^{2}\left(\varepsilon_{h}\right)+\left(\frac{1}{3}-c_{s}^{2}\left(\varepsilon_{h}\right)\right) \frac{X_{0} x+x^{2}}{X_{0} x+x^{2}+X^{\prime 2}}
$$

where $X_{0}=\sqrt{12} X^{\prime} c_{S}^{2}\left(\varepsilon_{h}\right)\left[X_{0}\right.$ Ratio], $x \equiv \ln \varepsilon / \varepsilon_{h}$, and $\varepsilon_{h}$ is the energy density at $T=165 \mathrm{MeV}$. The parameter $X_{0}$ Ratio determines how dramatically the speed of sound initially rises or falls at temperatures above $165 \mathrm{MeV}$ with a value of -1 corresponding to a minimum speed of sound equal to zero. The second parameter, $X^{\prime}$, determines the rate at which the speed of sound squared reaches its asymptotic value of $1 / 3$ at high temperatures. The prior for these parameters is assumed to be flat within the ranges of $-0.5<X^{\prime}<5$ and $-0.9<X_{0}$ Ratio $<2$ which allows for a wide range of possible equations of state as illustrated in Fig. 1.

The shear viscosity and its temperature dependence were also parameterized in the hydrodynamic stage of the model as

$$
\frac{\eta}{s}=\left(\frac{\eta}{s}\right)_{0}+\left(\frac{\partial \eta / s}{\partial T}\right) \ln \frac{T}{165 \mathrm{MeV}}
$$

where $\left(\frac{\eta}{s}\right)_{0}$ indicates the viscosity at a temperature of $165 \mathrm{MeV}$ and $\left(\frac{\partial \eta / s}{\partial T}\right)$ indicates the energy dependence of the viscosity. The viscosity at freeze out was allowed to vary in the range $[0,0.5]$ while the energy dependence varied from $[0,3.0]$. The resulting prior includes a wide range of possible shear viscosity values and energy dependencies that range from constant to unrealistically large. 


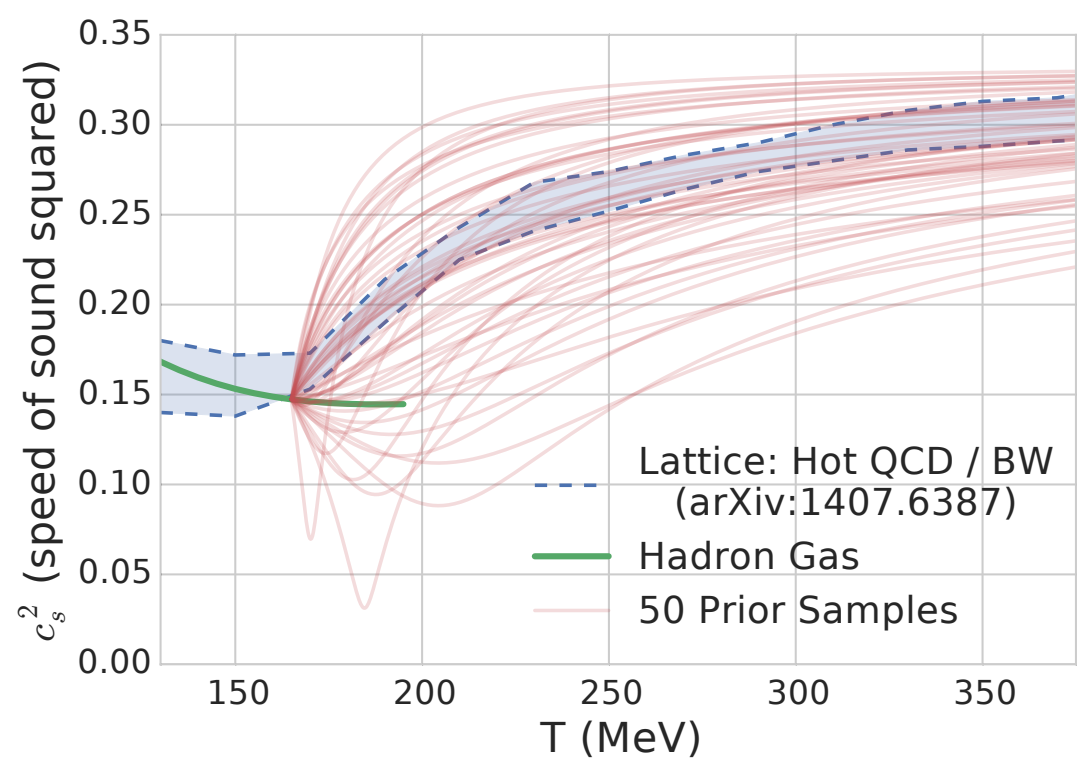

Figure 1: Random Equations of State Drawn From the Prior Distribution

These 50 random equations of state drawn from the prior distribution show the wide range of possibilities encompassed by the chosen parameterization. A range of possible values from lattice calculations is show in blue [5]. It is clear that equations of state that are either much stiffer or softer than the lattice result are allowed by the prior.

The remaining 10 parameters were used to describe the initial conditions before beginning the hydrodynamic evolution of the model. At each collision energy, three parameters varied the energy density normalization and described the saturation picture while a fourth described the initial flow as explained in [12]. A fifth parameter at each collision energy describes the initial anisotropy of the stress energy tensor and was varied such that longitudinal pressure, $T_{z z}$, could fall anywhere between zero and the pressure $P$.

A training set of 1000 model runs spread throughout the parameter space according to Latin Hypercube Sampling (LHS) was generated. A principle component analysis on the 30 observables was performed and all but the 14 most significant components, which captured over $99.9 \%$ of the total variance, were discarded. This dataset was then used to train a Gaussian process emulator which was employed to map out the posterior distribution over the parameter space using a MCMC as explained in [12]. Systematic model uncertainties of $6 \%$ were used in addition to the experimental uncertainties to account for the effects of missing physics in the model.

An additional set of 50 full model runs were evaluated at random points distributed according to this posterior distribution and the error in emulation was quantified and the emulator retrained. This validation step was repeated four times, resulting in a total of 1200 model runs, before the emulator's accuracy was found to converge to less than a few tenths of one unit when determining $\ln \mathscr{L}$. The results shown here are based on the posterior distribution obtained using the emulator trained with all 1200 model runs. 




Figure 2: Random Equations of State Drawn From the Posterior Distribution

These 50 random equations of state drawn from the posterior distribution show the degree to which the equation of state is constrained when compared to the prior samples in Fig. 1. The inferred equation of state has a slight preference to be softer than the lattice calculation [5] but they are entirely consistent.

\section{Results}

The described procedure resulted in significant constraints on the model parameters, including those used to describe the equation of state. The inferred equation of state is illustrated in Fig. 2 where 50 random samples from under the posterior were drawn in the same fashion as had been done with the prior in Fig. 1. By visually comparing these two plots it is clear that equations of state falling into the more extreme limits of being stiff or soft have been excluded from the posterior distribution. It appears that an equation of state falling significantly below the hadron gas value at $T=165 \mathrm{MeV}$ for any extended range is excluded and that it must rise with temperature. This result is highly consistent with the lattice calculation.

\section{Conclusions}

A parameterization of the equation of state of QCD matter has been statistically inferred from experimental measurements. The resulting distribution over possible equations of state suggests that the speed of sound squared gradually rises from the hadron gas value at $T=165 \mathrm{MeV}$. The result is very consistent with lattice calculations which lends credence to the supposition that the matter created in relativistic heavy-ion collisions behaves similarly to an equilibrated QGP. It additionally demonstrates that our model describes the dynamics of heavy-ion collisions well enough to permit the extraction of thermodynamic and transport properties of equilibrium QCD matter.

\section{Acknowledgement}

This work is supported by NSF grant NSF-0941373 and DOE grant DE-FG02-03ER41259. 


\section{References}

[1] J. Adams et al. [STAR Collaboration], Nucl. Phys. A 757, 102 (2005) [nucl-ex/0501009].

[2] J. Adams et al. [STAR Collaboration], Phys. Rev. Lett. 91, 172302 (2003) [nucl-ex/0305015].

[3] C. Ratti, R. Bellwied, M. Cristoforetti and M. Barbaro, Phys. Rev. D 85, 014004 (2012).

[4] A. Bazavov, T. Bhattacharya, M. Cheng, C. DeTar, H. T. Ding, S. Gottlieb, R. Gupta and P. Hegde et al., Phys. Rev. D 85, 054503 (2012) [arXiv:1111.1710 [hep-lat]].

[5] A. Bazavov et al. [HotQCD Collaboration], Phys. Rev. D 90, no. 9, 094503 (2014).

[6] L. Van Hove, Phys. Lett. B 118, 138 (1982).

[7] H. Sorge, Phys. Rev. Lett. 82, 2048 (1999) [nucl-th/9812057].

[8] H. Sorge, Phys. Rev. Lett. 78, 2309 (1997) [nucl-th/9610026].

[9] S. Pal and S. Pratt, Phys. Lett. B 578, 310 (2004) [nucl-th/0308077].

[10] S. Pratt, Phys. Rev. Lett. 102, 232301 (2009) [arXiv:0811.3363 [nucl-th]].

[11] U. W. Heinz, J. Phys. Conf. Ser. 455, 012044 (2013) [arXiv:1304.3634 [nucl-th]].

[12] J. Novak, K. Novak, S. Pratt, J. Vredevoogd, C. Coleman-Smith and R. Wolpert, Phys. Rev. C 89, 034917 (2014) [arXiv:1303.5769 [nucl-th]]. 